\title{
REALIDADES ANTROPOLÓGICAS
}

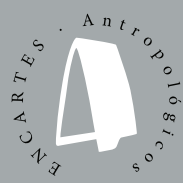

ROLES DE GÉNERO EN LA GULTURA TOTONAGA DENTRO DE LA PRODUGGIÓN DE GAFÉ. EL CASO DE ZONGOZOTLA

TOTONACO GENDER ROLES IN GOFFEE PRODUGTION: ZONGOZOTLA

\author{
Maritel Yanes Pérez* \\ Luis Roberto Canto Valdés**
}

Resumen: En este estudio analizamos los roles de género que asumen algunos hombres y mujeres de origen totonaco que se dedican al cultivo del café en el municipio de Zongozotla, en la Sierra Norte del estado de Puebla, para ver qué prácticas tradicionales se siguen observando y evidenciar aquellos valores y creencias que se han modernizado. Utilizamos la técnica de observación participante, realizamos quince entrevistas a mujeres totonacohablantes acompañadas de sus esposos. Interpretamos nuestros resultados desde una perspectiva de género, pero respetando la cosmovisión indígena de las y los entrevistados. Encontramos patrones de conducta tradicionales y algunos elementos que nos obligan a repensar el género desde la mirada de las personas originarias.

Palabras claves: género, totonacos, pueblos originarios, café, cosmovisión.

\section{TOTONAGO GENDER ROLES IN COFFEE PRODUGTION: ZONGOZOTLA}

Abstract: The study analyzes gender roles that certain Totonaco-origin men and women who raise coffee in the Zongozotla municipal jurisdiction, in Puebla's Sierra Norte, take on, as a means of seeing which traditional practices are still being observed as well as to shed light on values and beliefs that have modernized. To reach our goal, we used the participant observation technique and undertook fifteen interviews with Totonaco-speaking women, in the company of their

\footnotetext{
* El Colegio de México.

** Centro de Investigación y de Estudios de Antropología Social (CIESAS).
}

ISSN: 2594-2999, Bajo licencia Creative Commons

ENCARTES antropológicos 2 • septiembre 2018-marzo 2019, pp. 173-188

Recepción: 25 de enero de 2018 • Aceptación: 2 de abril de 2018

http://www.encartesantropologicos.mx

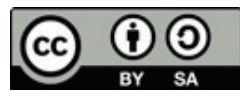


husbands. We interpret the outcomes from a gender-based perspective while respecting indigenous worldviews of all interviewees, women and men alike. We discovered traditional behavior patterns and certain elements that oblige us to re-think gender from first peoples' perspectives.

Key words: gender, Totonaco, first peoples, coffee, worldviews.

\section{I}

NTRODUGCIÓN

A lo largo del desarrollo de la sociedad las personas aprendieron, a través del proceso de socialización, cómo comportarse según fueran hombres o mujeres. Esta diferenciación abarca normas de comportamiento, actitudes, valores, tareas, entre otras cosas. Sin embargo, históricamente se ha privilegiado lo masculino sobre lo femenino.

Los sistemas de sexo/género han sido el objeto de estudio más amplio para comprender y explicar la subordinación femenina y la dominación masculina. La categoría de género reconoce una dimensión de la desigualdad social, diferente de la dimensión económica, de las teorías de las clases y de la estratificación social (De Barbieri, 1993).

El feminismo poscolonial expone que hay que estudiar las situaciones particulares con tal de proporcionar explicaciones a partir de las especificidades contextualizadas (Brunet y Pizzi, 2011). No existe una forma única de ser hombre o de ser mujer, ya que sus actividades, sus limitaciones y sus posibilidades varían de una cultura a otra (Lamas, 1986). No podríamos analizar el género sin contextualizarlo en un tiempo concreto, en un lugar determinado y en una sociedad dada, debido a que sus características vienen establecidas por las pautas culturales, sus normas, valores, y la división por género del trabajo que establece cada sociedad (Alberti, 1999).

Debido a lo anterior, desde la década de los setenta, y con mayor intensidad en los ochenta, se realizaron estudios incorporando la categoría del género con el objeto de describir cómo se construyen y se conceptualizan las relaciones entre los hombres y las mujeres dentro de los sistemas de valores y creencias de los grupos étnicos (Sánchez y Goldsmith, 2000).

En los pueblos originarios la tradición y la modernidad son dos constantes que interactúan permanentemente. La modernidad puede interpretarse como un concepto puramente relativo cuyo sentido reside en ser lo opuesto de la "tradición", o, más exactamente, la "no tradición". La 
modernidad es entendida como un factor externo que afectaría las culturas nativas tradicionales (Pitrach y Gemma, 2012).

De tal manera, las mujeres y los hombres indígenas dan valor a elementos de la modernidad, adquiriendo unos y rechazando otros. La pertenencia a un grupo étnico define al género; las mujeres se incluyen en una etnia y desde sus referentes simbólicos entienden a los otros, al mundo y a sí mismas (Alberti, 1999).

En este estudio analizamos los roles de género que asumen algunos hombres y mujeres de origen totonaco que se dedican al cultivo del café en un municipio de la Sierra Norte del estado de Puebla, para ver qué prácticas tradicionales se siguen observando y evidenciar aquellos valores y creencias que se han modernizado, así como los factores que están modificándolos.

\section{Métodos Y TÉGniCAS DE INVESTIGACIÓN}

El trabajo de campo tuvo lugar en el primer semestre del 2016, en el marco de las jornadas de vinculación con la comunidad de la Universidad Intercultural del Estado de Puebla (UIEP) en el pueblo de Zongozotla. La brigada estuvo integrada por las y los estudiantes de la UIEP Ambrosio Juárez Esteban, Alejandra Vázquez Guzmán, Alfredo Bautista Juárez y Luz Yaneth Esteban Cruz, todos ellos bilingües (hablantes de totonaco y español) bajo la dirección del doctor Luis Roberto Canto. Los datos fueron recabados a través de la técnica de observación participante. Se realizaron en total quince entrevistas a mujeres en lengua totonaca. La transcripción y traducción de dichas entrevistas fueron realizadas por Luz Yaneth Esteban, estudiante de lengua y cultura.

El rango de edad de estas mujeres oscilaba entre los 40 y los 50 años. Los testimonios fueron tomados siguiendo a las mujeres hacía sus centros de trabajo. Muchas de ellas nos permitieron repetir la ruta que día a día siguen.

Las narrativas de las mujeres fueron interpretadas desde la perspectiva de género considerando su cosmovisión, su historia y sus tradiciones nacionales, comunitarias y familiares. La categoría de género es adecuada para analizar y comprender la condición femenina y la situación de las mujeres, y lo es también para observar la condición masculina y la situación vital de los hombres. Es decir, el género permite entender a cualquier sujeto social cuya construcción se apoye en la significación social de su 
cuerpo sexuado con la carga de deberes y prohibiciones asignados para vivir, y en la especialización vital a través de la sexualidad (Lagarde, 1996).

En cuanto al contexto geográfico, el municipio de Zongozotla colinda al norte con Zapotitlán de Méndez y Camocautla, al este con Zapotitlán de Méndez y Huitzilan de Serdán, al sur con Cuautempan y al oeste con Tepango de Rodríguez y Teptzintla (Enciclopedia de los Municipios y Delegaciones de México, 2018). Es uno de los 217 municipios poblanos, un territorio donde se siembra y cosecha principalmente el café. Esta zona es fría los 365 días del año. Las faldas de las montañas, y las mismas montañas, son "el parador" de los cultivos. También se produce durazno, maíz y frijol. Su principal actividad económica es la agricultura (Enciclopedia de los Municipios y Delegaciones de México, 2018). Las corrientes del río Zempoala también surcan este municipio. El frío es más intenso durante el invierno y las lluvias son comunes en el municipio durante buena parte del año, especialmente a partir de agosto. Las calles de la comunidad son variables, pues algunas están en picada y otras en pendientes muy altas. Durante las lluvias, el río Zempoala, que cruza varios municipios poblanos del totonacapan, suele crecer.

Según el Consejo Nacional de Evaluación y Política de Desarrollo (CONEVAL, 2010), la población total de este municipio está compuesta por 2258 hombres (49\%) y 2341 mujeres (51\%), y solamente 721 personas hablan totonaco $(16 \%)$. De su población, $56.5 \%$ vive en condiciones de pobreza moderada, mientras que $29.9 \%$ está en una situación de pobreza extrema, y $11.6 \%$ es vulnerable por las carencias sociales. En cuanto a la escolaridad, el grado promedio de la población de 15 años o más en el municipio de Zongozotla era de 5.9 en el 2010, frente al grado promedio de escolaridad de 8 en la entidad (CONEval, 2010).

En promedio, el tamaño de los hogares es de 4.5 integrantes (CONEVAL, 2010). En el 2010, el porcentaje de individuos que reportó habitar en viviendas con mala calidad de materiales y espacio insuficiente fue de 23.4\% (1 019 personas) (CONEval, 2010). En nuestro trabajo de campo antropológico nos percatamos que las viviendas en general han podido tener un techo y piso de concreto gracias a los programas "piso digno" y "techo digno" del gobierno poblano en los últimos siete años, pero aún queda mucho por hacer.

La vida transcurre allí con pocos sobresaltos, casi todas las personas se conocen en la comunidad. La presidencia municipal tiene un altavoz 
desde donde se dan avisos o llamados en la lengua originaria del lugar: tutunakú, y también en español.

En la plaza de la comunidad transcurre parte de la vida de las y los habitantes porque es el principal nicho de sociabilización, las distintas generaciones de la población de Zongozotla acuden allí por diferentes razones (una de ellas es la señal de internet que les permite a las y los jóvenes entrar al "ciberespacio" con sus celulares). La iglesia agrupa a las y los católicos, sin embargo, también existe la pluralidad de creencias cristianas. Las escenificaciones culturales son comunes en las escuelas y en la plaza principal.

\section{LOS HOMBres y LAS MUJERES. LA RUTINA DE CADA DÍA}

Según lo observado, en el trabajo de campo, los hombres van delante de las mujeres por doquier, dejando a la mujer detrás de ellos en el camino que repiten tanto de ida como de vuelta. Este hecho fue perceptible donde quiera que vimos a las mujeres.

La única forma de saber más allá de lo observado en el diario de campo es complementarlo con entrevistas a las informantes. Las mujeres entrevistadas coincidieron de manera general en que "el hombre debe de ir delante para abrir el camino, ése es su lugar, así nos protegemos".

Lo que las mujeres no nos dijeron en las entrevistas es que sobre todos los peligros que los hombres tienen que sortear al abrir paso el más común es la presencia de serpientes venenosas, como la nauyaca y la coralillo, para cuyo veneno no hay un tratamiento cercano salvo en los hospitales de Puebla.

Una mujer que se identificó como doña Mary ${ }^{1}$ fue encontrada caminando con su esposo cerca de la salida del municipio por donde está el manantial de agua dulce próximo al río. La mujer fue interceptada en el camino; al momento le preguntamos si podríamos hacerle una entrevista, antes de contestarnos miró a su marido, quien bajó la cabeza meneando el sombrero hacía abajo y luego hacía arriba en señal de aprobación.

Ella, al igual que las otras mujeres, nos contestó lo siguiente: "Caminamos a la par con nuestro hombre, porque él es el sustento de nuestro hogar".

\footnotetext{
${ }^{1}$ Todos los nombres fueron cambiados con la finalidad de proteger la identidad de las y los entrevistados.
} 
Doña Mary, como las otras mujeres ubicadas en las orillas del río, estaba recogiendo leña para llevar sobre sus hombros, porque las mulas y los burros no pueden entrar a ese difícil terreno, e indicó:

Sin el hombre la familia no funciona... por eso me levanto temprano para calentar el café, y a la mesa se le deja el pan caliente, frijoles y un poco de huevo revuelto... así es como es el amanecer de cada día para nosotros.

En nuestra investigación observamos que la labor del campo inicia muy temprano por la mañana; las mujeres deben ponerse de pie mucho antes de que salga el sol y de que los hombres despierten, ya que el café debe de estar listo junto con el primer alimento.

Las mujeres tienen un rol en la producción del café, ya que no solamente acompañan a sus maridos, sino que también los ayudan a labrar la tierra húmeda y a depositar en ella la semilla que al cabo de un tiempo germinará y dará producto. Las mujeres laboran a la par de los hombres, no les dejan todo el trabajo; en efecto, así como ellos cargan la leña sobre la espalda, ellas también lo hacen. De la misma forma en que ellos toman el arado y el machete para labrar la tierra del monte, ellas llevan a cabo lo mismo.

Las mujeres perciben que la mayor parte del trabajo recae en el hombre, quien de forma tradicional dentro de este pueblo del Totonacapan tiene el rol de proveer y proteger el hogar. Sin embargo, en nuestro trabajo de campo se pudo observar que la mujer también participa en las faenas del campo junto con el hombre, quien ciertamente realiza la mayor parte de la labor, pero no toda. Las mujeres no ven todo ello como una carga; lo consideran como parte de su colaboración en pro de la familia. Además de esto, las mujeres están más enfocadas a la crianza de las y los niños y también en las labores del hogar. Ellas, por tanto, deben asumir varios roles que tienen que ir alternando.

Las y los niños suelen crecer viendo a sus padres labrar la tierra, pero ya jóvenes abandonan el hogar por diversas razones. Dos de las mujeres indicaron que sus hijos e hijas ya no viven con ellas:

Ellos crecieron... se fueron, olvidaron sus raíces, viven en la ciudad porque ya son profesionistas, uno es doctor, y los otros dos son ingenieros... mis dos hijas se metieron con hombres casados y ya formaron su familia. 
La añoranza es un sentimiento que puede verse en los testimonios de estas mujeres, que miran hacia abajo para después perder la mirada en el cielo. Ellas saben que sus hijas e hijos ya se fueron, que formaron una familia y que hasta se olvidaron de sus raíces.

La migración es un fenómeno humano muy común en todas las sociedades, a menudo se relaciona con el partir de las hijas y los hijos hacia nuevos horizontes, que casi siempre tienen que ver con hallar oportunidades laborales que no hay dentro de la comunidad. Con añoranza, el hombre y la mujer, muy arriba de la montaña, dijeron ser los dueños legales de dicho terreno. Una mujer manifestó:

Nuestros hijos aquí jugaban cuando tú sembrabas café y durazno con tus hermanos, sus juguetes eran las ramas, la leña y los instrumentos de labranza, todos querían ser como su papá y su mamá, pero la escuela cambió todo esto. Estudiaron y se fueron, es la ley de la vida.

Es por esta razón que, en la actualidad, las escuelas son vistas con cierto recelo por algunas de las mujeres entrevistadas, ya que con el estudio muchos de sus hijas e hijos cambiaron de mentalidad y vieron al campo $\mathrm{y}$ a la vida rural con menosprecio, prefiriendo lo occidental junto con la moda. El nexo que existe en la familia se diluye con la distancia, y con el tiempo las visitas de las hijas y los hijos a los padres se pierden.

Otro fenómeno que se observa cuando las y los hijos se van del pueblo de origen es el olvido de la lengua originaria, como se ve en los testimonios de dos mujeres entrevistadas:

Ya se olvidaron de hablar como sus abuelos, así como nosotros en nuestra lengua.

Ellos ya no hablan a su madre en nuestra lengua, nos llegan a decir "mother". Mi hijo se fue a las "gringolandias" (Estados Unidos).

La migración, según algunos de los relatos, siempre lleva a las hijas y los hijos a la capital poblana o a la capital del país. Quienes no estudiaron o no tienen tierra u oportunidad para recibirla por herencia dejan el país y se van a los Estados Unidos a buscar otras oportunidades y no cuentan con documentos legales para acreditar su estancia en el vecino país. Al respecto, una de nuestras entrevistadas dijo lo siguiente: 
Ya no recuerdo mucho su rostro... miro mucho su foto vieja de cuando muchachillo para recordarlo... mi hijo es bueno y que el cielo lo cuide... no sé mucho de él, sé que está vivo porque llega un dinerito que no me ha dejado de mandar... me lo aumenta el diez de mayo... así sé que está vivo... no sé qué clase de peligros enfrente mi niño... pero sé que le va bien porque lo eduqué con mucha disciplina para formar hombre de bien como con sus otros hermanos.

Durante las entrevistas, los hombres continuaron laborando pero estaban al pendiente de sus esposas, y en ocasiones intervenían en la entrevista, no para interrumpirla sino más bien para apuntalar el relato de las mujeres. El contacto visual, según pudimos observar, es muy importante entre ellos. Al finalizar, las mujeres se recostaban sobre la tierra para ceñir sus frentes con un lazo trenzado que les ayudaba a ir cuesta abajo con toda la leña que llevaban a la espalda. Los hombres también llevaban una carga similar, más los aperos de labranza.

Todas nuestras entrevistas terminaban más o menos de la misma forma: la vuelta al hogar con la leña, con el cuerpo fatigado a causa de las labores agrícolas que comienzan desde que el sol muestra sus primeros rayos del alba. También el regreso a casa comienza cuando el Astro Rey se sitúa por completo sobre el cielo. Un descanso es normal, y después viene el almuerzo.

\section{LA VIOLENCIA EN GASA}

Entre los hombres y las mujeres, dentro de las relaciones afectivas, siempre se dan dificultades. En este sentido, las mujeres, en su rol de esposas, muchas veces tienen que ser el apoyo para los maridos, quienes en ocasiones causan problemas dentro y fuera de la casa.

Seis de las mujeres entrevistadas señalaron explícitamente sufrir violencia en sus hogares por parte de sus esposos cuando se encuentran en estado de ebriedad. Ellas no se quejan y guardan silencio, y no se atreven a denunciarlos para evitar los chismes del pueblo; por tanto, la violencia ocurrida en la casa no suele por lo general llegar al conocimiento de las autoridades correspondientes de Zongozotla. Una mujer que fue ubicada en los bajos de la montaña dijo algo muy revelador: 
El marido sí nos pega, nos da bofetadas o nos da con el sombrero, pero si lo denunciamos puede ir a la cárcel, y si va a la cárcel se pierde el sustento de nuestra familia, ¿qué haremos nosotras sin nuestro hombre?, a veces tiene otra mujer, y lo sabemos, pero mejor no decimos nada porque también nos puede reprender... no perdemos nuestro lugar como la mujer legítima ante los demás... la otra es la otra y a nosotras la dicha en el altar ante los ojos de Dios como juez último.

Esta entrevistada indicó que este tipo de problemas se resuelve dentro de la familia para que no tome otras dimensiones. Es decir, los hermanos y el padre de la mujer hablan con el marido para pedirle que no se comporte así con ella y que le dé un trato más cordial. El hombre suele asimilar esto y cambia su conducta, hasta que de nuevo el "agua del viñedo de la caña" se tope con su paladar y se repitan las acciones violentas.

El hombre sí ejerce violencia hacia su pareja, pero también la protege, ya que suele ir delante. Los hombres son quienes afrontan los peligros que la naturaleza oculta, como las serpientes, y los ladrones, y con el machete ceñido a la cintura van delante en el camino para hacerles frente.

Los hombres son los encargados de disciplinar a los hijos, y en las riñas, indicaron, tienen "la sangre caliente", ya que siempre suelen estar prestos a responder con agresividad ante un intruso o invasión; sobre todo cuando el alcohol les acompaña.

Las mujeres entrevistadas dicen que, entre hombres, la fuerza suele ser un recurso importante para decir "quién es el que manda", y también es vital para elegir pareja:

Cuánta leña levantan, cuánto pueden cargar, y qué pueden hacer... eso determina muchas cosas para nosotras... y también para las que tienen una relación a la par con nuestros hombres... los hijos se conocen y lo saben, pero no dicen nada para no aumentar nuestra pena ni la suya. El hombre no será juzgado por nosotras. (Lantla xlilhuwa sakkgoy xtasakgnikan, lantla xlilhuwa kukanankgoy, chu tuku katsini tlaway... lhuwa tuku kinkalimasiyaniyan... chu nachuna ukxilhkgoy wantiku xakgatlikgoy kilakchixkuwinkan... lalakgapaskgoy kamanin chu katsikgoy kaxman pi nitu wankgoy xpalakata ni nakinkamakgalipuwankgoyan chu ni nalipuwankgoy. Ni akxniku ktilichuwinaw chixku). 
Tenemos que señalar que entre traducción y traslación de una lengua a otra se pudo notar un hecho importante. La palabra juzgar se refiere, en español, a emitir una sentencia. Pero en tutunakú no existe una equivalencia como tal, lo único que se dice es que no pueden decir, de la boca para afuera, nada que evalúe la conducta del hombre.

Cabe mencionar que dicho testimonio es también revelador ya que muestra que las mujeres de esta comunidad esperan que los hombres sean fuertes, un evidente símbolo de masculinidad, y esto le dará al varón un cierto estatus ante otras mujeres.

Por otra parte, también nos preguntamos si las mujeres ejercían violencia hacia sus parejas. Después de platicar con una mujer en las orillas del río y lejos de la presencia del hombre, captamos información muy interesante que nos puede ayudar a entender que las mujeres sí aplican la violencia, pero de manera diferente.

La violencia ejercida por las mujeres, según el testimonio de tres de las entrevistadas, es una revancha de lo que sufren, pero pidieron que esta información no se grabara, por lo que tomamos notas de campo para rescatar parte de lo que decían.

Las mujeres indicaron que en ocasiones la comida es mal hecha a propósito, para causar malestar al estómago del hombre, o bien esconden sus cosas para enojarlo y hacerle perder el tiempo. Otro recurso es el de echar a perder la ropa o algún otro objeto que sea del gusto del hombre. Según el testimonio de una de nuestras entrevistadas, el alcoholismo de los hombres también les brinda otra posibilidad:

Cuando los hombres están borrachos no saben qué pasa a su alrededor, entonces les decimos insultos... también les jalamos el pelo o les pegamos en la cara... y al día siguiente ellos creen que esto es por la cruda.

De lo anterior se puede desprender la idea de que dentro del hogar se da toda una serie de hechos que involucran violencia entre ambas partes. Un señor manifestó que la violencia "es la sal de una relación... le da sabor" (Wa xli maskgokgenat uyma talakxtumit... maxki liskamat).

La diferencia en el ejercicio de la violencia entre hombres y mujeres es que algunas de ellas también ejercen violencia, pero lo hacen de forma diferente, de manera silenciosa e inadvertida. 


\section{Discusión}

De acuerdo con nuestros resultados, encontramos diferentes elementos que deben recuperarse desde una perspectiva de género. A la par, trataremos de analizarlos desde una visión que respete la cosmovisión indígena totonaca; como bien menciona Díaz (2014), hilar las categorías de etnia y género busca develar el contexto simbólico de las particularidades y los procesos de construcción y significación indígenas, además de revisar sus trascendencias tanto para las mujeres como para los hombres (Díaz, 2014).

El papel del hombre en Zongozotla sigue siendo el tradicional, pues es considerado proveedor y protector del hogar y la familia. Mientras más fuerza tenga, más hombre es. Un hecho presente en la comunidad es el alcoholismo de los hombres, "pues ellos cuándo están borrachos no saben lo que pasa a su alrededor". Éste es un factor que contribuye al ejercicio de la violencia hacia las mujeres y una constante en las comunidades indígenas (Valladares, 2007).

Una de las acciones contra la violencia es que los hermanos y el padre de la mujer hagan ver al marido su mala conducta para que la corrija. Esto confirma que el sistema de patriarcado sigue vigente. El hombre es violento también en las riñas, ante los intrusos y los peligros, sobre todo bajo los efectos del alcohol.

Sin embargo, en el ejercicio de la masculinidad totonaca, en este pueblo originario el hombre arriesga su integridad física (Secretaría de Salud, 2006), pero por el bienestar de su familia. Ellos van al frente en el camino no para denigrar a las mujeres sino para enfrentar los peligros al internarse en las montañas.

Por su parte, la mujer sigue siendo por lo general sumisa, requiere de la aprobación y del acompañamiento del esposo a donde quiera que vaya. Las labores del hogar corresponden a la mujer, esto coincide con diversas investigaciones que se han hecho en otras regiones cafetaleras (Cárcamo et al., 2010). Nuestras entrevistadas interpretan su labor en el hogar como una contribución positiva para su buen funcionamiento. El hombre tiene que trabajar para llevar el sustento del hogar y la mujer debe cocinarle para que vaya alimentado al campo.

La participación de las mujeres en la producción del café es un tema escasamente explorado en México (Vargas, 2007). Las mujeres de Zongozotla no visualizan su participación en la elaboración del café como tal, 
pero ellas también labran la tierra y siembran a la par que sus esposos, y tienen una participación activa dentro de las labores del campo; allí tienen presencia desde antaño, y no sólo como compañeras, sino también como esposas y madres, porque la familia completa participa en el proceso de la producción.

Ahora bien, observamos que las mujeres tienen que desempeñar diferentes roles a lo largo de cada día, y repetirán esta rutina durante toda su vida, aceptando los cambios que el tiempo les impone. Un rol que solo dejarán a causa de la viudez es el de ser esposas, pues el de madres lo tendrán todo el tiempo hasta que las y los hijos migren a otro lugar en búsqueda de trabajo o con la esperanza de una vida mejor.

La migración impacta no sólo a estas personas que se quedaron en sus pueblos sino a las que se fueron (Klein y Vázquez, 2013). Es interesante la percepción de algunas de las madres sobre sus hijas e hijos migrantes, ellas sienten que ya se olvidaron de sus raíces.

Otro de los factores que influyen en el cambio de comportamiento de las hijas y los hijos es la educación, ya que se valoran más las costumbres que vienen de fuera; no sólo abandonan el campo, sino que olvidan a sus familias y también dejan a un lado su lengua originaria.

En efecto, en otras investigaciones se ha confirmado que los principales factores que influyen en las nuevas identidades juveniles son la migración, la educación y los medios de comunicación (Gavilánez y Pilaguano, 2015).

Con respecto a la violencia ejercida por sus maridos, las mujeres no los denuncian para evitar estar en los comentarios del pueblo. El silencio ante la violencia es una práctica común en muchas comunidades del Totonacapan, como se ha documentado en otros estudios; en la Sierra Norte de Puebla el modelo genérico y familiar le confiere al jefe la autoridad para "disciplinar" a los demás miembros de la familia, castigándolos físicamente cuando no cumplen con las obligaciones de servicio y obediencia que les asigna el modelo, de modo que los golpes son vistos como una prerrogativa legítima de los padres y los maridos (González, 2009).

Algunas mujeres manifestaron que si el marido va a la cárcel se pierde el sustento de la familia. Las infidelidades son perdonadas con el fin de mantener la familia, mientras el hombre garantice el sustento del hogar y siga siendo fuerte, a las mujeres parece no importarles "compartir" a sus esposos si ellas son las esposas legítimas. 
Por otra parte, algunas de las mujeres entrevistadas también ejercen violencia contra los hombres, al parecer como venganza por los maltratos recibidos. Hacen mal la comida, esconden cosas para hacerlos enojar o incluso los golpean aprovechándose de ellos cuando están borrachos para jalarles el pelo o golpearles en la cara. Al parecer, las prácticas violentas de las mujeres responden a contextos dificiles, y muchas veces son resultado de una violencia de género recurrente en la familia (Beltrán, 2012).

\section{Conclusiones}

En esta investigación analizamos los roles de género que asumen algunos hombres y mujeres de origen totonaco que se dedican al cultivo del café en el municipio de Zongozotla, Puebla, para ver qué prácticas tradicionales se siguen observando y evidenciar aquellos valores y creencias que se han modernizado, así como los factores que están modificándolos. Observamos que las mujeres y los hombres totonacos que se dedican a la producción del café adoptan diferentes roles de género. Lo cultural cobra vital importancia dentro de los roles que se espera acate cada sexo, por lo que, tanto hombre como mujer, al crecer saben qué es lo que tienen que hacer.

El hombre sigue conservando el papel de proveedor y protector del hogar. Es interesante analizar la percepción de la fuerza de los hombres por parte de las mujeres. Esa fuerza es la que las atrae, pero al mismo tiempo es la que las somete a los golpes de sus maridos cuando se encuentran bajo los efectos del alcohol. Al respecto, no visualizamos ninguna acción por parte de las respectivas autoridades ni de la sociedad civil para atender el alto consumo de alcohol que existe en las comunidades del Totonacapan, por lo que se recomienda abordar este problema de salud pública.

Otro fenómeno que falta erradicar es el ejercicio de la violencia contra la mujer, que al parecer en este caso está directamente relacionado con el alcohol, pues las mujeres declararon que sus maridos solamente las golpean cuando están borrachos. Un dato interesante de resaltar es que los hombres caminan delante de las mujeres por una razón: para protegerlas de los peligros de las montañas; lo hacen porque para ellos la mujer es muy importante, por lo que la deben proteger frente a cualquier amenaza. $\mathrm{Al}$ respecto, ellas enfatizaron que eso es lo que se espera de un hombre. Esta práctica es diferente del deber ser del pensamiento occidental, donde ambos caminan a la par enfrentando los mismos peligros al mismo tiempo. 
Por otra parte, la mujer representa la sumisión. Asume en su vida diaria el papel de madre, esposa, trabajadora doméstica y agricultora. Su participación en la producción del café es activa a pesar de que ella misma minimiza su actuación.

Las mujeres de Zongozotla sufren violencia por parte de sus esposos, pero es un secreto. La carga social impuesta a la mujer es significativa: no se denuncia porque se teme al juicio de la comunidad y a la pérdida de la familia. Algunas de las mujeres han creado medios de venganza contra sus maridos, confirmando que la violencia crea más violencia. Los medios para ejecutarla son diferentes, casi imperceptibles, "silenciosos". Consideramos que estos matices deben de ser tomados en cuenta al momento de evaluar los patrones culturales. Surge la interrogante: ¿las hijas y los hijos también son tocados por un sistema de violencia gestado dentro de la cosmovisión totonaca?

Distinguimos dos factores que están modificando las interacciones dentro de las familias, y por supuesto las relaciones de género: la migración y la educación.

A partir de estos hallazgos exhortamos a que se sigan desarrollando más investigaciones que analicen y profundicen en las relaciones de género de personas de origen indígena y los factores que están modificando los vínculos.

\section{$\Delta$}

\section{BIBLIOGRAFÍA}

Alberti Manzanares, Pilar (1999). "La identidad de género y etnia. Un modelo de análisis", en Nueva Antropología, junio, pp. 105-130.

Barbieri, Teresita de (1993). "Sobre la categoría género: una introducción teórico-metodológica”, en Debates en Sociología, núm. 18, pp. 2-19.

Beltrán Gálvez, María (2012). "La otra cara de la moneda: mujeres que practican violencia”, en Revista Punto Género, 0 (2), pp. 71 - 92.

Brunet, Ignasi y Alejandro Pizzi (2011). "Empobrecimiento y exclusión de género en España", en Actores del desarrollo en la primera mitad del siglo XXI: Innovación y Cambio, Montevideo, Ateneo Ayuí de Ciencias Sociales, pp. 594-615.

Cárcamo Toalá, Naima Jazíbi, Verónica Vázquez García, Emma Zapata Martelo y Austreberta Nazar Beutelspacher (2010). "Género, traba- 
jo y organización: mujeres cafetaleras de la Unión de Productores Orgánicos San Isidro Siltepec, Chiapas", en Estudios Sociales 18(36), 155-176. Recuperado de: http://www.scielo.org.mx/scielo.php?script=sci_arttext\&pid=S0188-45572010000200007\&lng=es\&nr$\mathrm{m}=$ iso $>$. ISSN 0188-4557.

Coneval (2010), "Municipio de Zongozotla, Puebla". Recuperado de https://www.gob.mx/cms/uploads/attachment/file/40690/Puebla_215.pdf

Díaz Cervantes, Rufino (2014). "La perspectiva de género en la comprensión de la masculinidad y la sobrevivencia indígena en México", en Agricultura, sociedad y desarrollo, 11(3), pp. 359-378.

Enciclopedia de los Municipios y Delegaciones de México (2018). "Zongozotla". Recuperado de http://www.inafed.gob.mx/work/enciclopedia/ EMM21puebla/municipios/21215a.html

Gavilánez Chusin, Willian Neptali y Hugo Fabién Pilaguano Pilaguano (2015). "Cambios identitarios de los jóvenes indígenas de Cotopaxi: estudio de caso en la comunidad de San Antonio de Chaupi, barrio Loma de Panecillo". Tesis de Licenciatura, Universidad Salesiana de Ecuador.

González Montes, Soledad (2009). "Violencia contra las mujeres, derechos y ciudadanía en contextos rurales e indígenas de México", en Convergencia. Revista de Ciencias Sociales, 16 (50), 165-185.

Herrera Santi, Patricia (2000). "Rol de género y funcionamiento familiar", en Revista Cubana de Medicina General Integral, 16(6), pp. 568-573. Klein, Alejandro y Érika Vázquez-Flores (2013). "Los roles de género de algunas mujeres indígenas mexicanas desde los procesos migratorios y generacionales", en Fournal of Behavior, Health E Social Issues, mayo-octubre, pp. 25-39.

Lagarde, Marcela (1996). "El género", en Género y feminismo. Desarrollo humano y democracia, Madrid, Ed. horas y HORAs, pp. 13-38.

Lamas, Martha (1986). "La antropología feminista y la categoría 'género"”, en Nueva Antropología, vol. III, núm. 30, pp. 173-198.

Pitrach, Pedro y Gemma Orobitg (2012). "Prefacio", en Pedro Pitarch y Gemma Orobitg (ed.), Modernidades indígenas. Madrid: Iberoamericana Vervuert y Bonilla Artigas Editores, pp. 9-19.

Sánchez Gómez, Martha Judith y Mary Goldsmith (2000). "Reflexiones en torno a la identidad étnica y genérica. Estudios sobre las mujeres 
indígenas en México", en Política y Cultura, núm. 14, 2000, pp. 61-88. Secretaría de Salud (2006). "Boletín de práctica médica efectiva". Sección: factores poblacionales de riesgo para las ITS. Abril, México: SSA. INSP. Valladares, Laura (2007). "Transgredir y construir una vida digna: el encuentro de la doctrina de los derechos humanos entre las mujeres indígenas en México", en E. Olavarría (coord.) Simbolismo y poder, uAm/Porrúa, México.

Vargas Vencis, Perla (2007). "Mujeres cafetaleras y producción de café orgánico en Chiapas”, en El Cotidiano, vol. 22, núm. 142, marzo-abril, pp. 74-83.

Maritel Yanes Pérez es licenciada en Derecho por la Universidad Juárez Autónoma de Tabasco, maestra en Demografía por El Colegio de la Frontera Norte, doctora en Estudios de Población por El Colegio de México. Cátedras ConacyT comisionada en El Colegio de la Frontera Sur Unidad Villahermosa. Temas de especialización: violencia, género, derechos humanos y pueblos originarios.

Luis Roberto Canto Valdés es licenciado en Ciencias Antropológicas por la Universidad Autónoma de Yucatán, maestro y doctor en Historia por el Centro de Investigación de Estudios de Antropología Social (CIESAS) Peninsular. Docente e investigador de tiempo completo de la Universidad Intercultural del Estado de Puebla. Temas de especialización: criminalidad, medicina alternativa, pueblos originarios, y antropología de la salud. 
Ángela Renée de la Torre Castellanos

Directora de Encartes antropológicos

Arthur Temporal Ventura

Editor

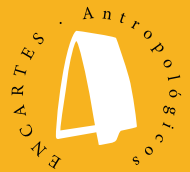

Verónica Segovia González

Diseño y formación

Cecilia Palomar Verea

María Palomar Verea

Corrección

Saúl Justino Prieto Mendoza

Difusión

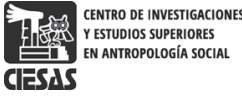

Encartes cuenta con el apoyo de El Colegio de la Frontera Norte.

Equipo de coordinación editorial

Renée de la Torre Castellanos Directora de Encartes antropológicos - María Eugenia de la O Martínez CIESAS -Occidente - Joel Pedraza Mandujano CIESAS -Occidente - Santiago Bastos Amigo CIESAS -Occidente Manuela Camus Bergareche Universidad de Guadalajara - Alejandra Navarro Smith ITESO - Luis Escala Rabadán El COLEF

\section{Comité editorial}

Agustín Escobar Latapí Director general de CIESAS - Alberto Hernández Hernández Presidente de El colef Andrés Fábregas Puig CIESAS -Occidente - Dulce Mariana Gómez Salinas Subdirectora del departamento de publicaciones de CIESAS - Érika Moreno Páez Coordinadora del departamento de publicaciones de El colef José Manuel Valenzuela Arce El colef - Luz María Mohar Betancourt CIESAS -Ciudad de México - Ricardo Pérez Monfort CIESAS -Ciudad de México - Sévérine Durin Popy CIESAS -Noreste C Carlos Yuri Flores Arenales Universidad Autónoma del Estado de Morelos - Sarah Corona Berkin DECS /Universidad de Guadalajara Norma Iglesias Prieto San Diego State University - Camilo Contreras Delgado El colef

Cuerpo académico asesor

Alejandro Frigerio

Universidad Católica

Argentina-Buenos Aires

Alejandro Grimson

USAM -Buenos Aires

Alexandrine Boudreault-Fournier

University of Victoria-Victoria

Carlo A. Cubero

Tallinn University-Tallin

Carlo Fausto

UfRJ -Rio de Janeiro

Carmen Guarini

UBA -Buenos Aires

Caroline Perré

Centro de Estudios Mexicanos y

Centroamericanos-Ciudad de

México

Clarice Ehlers Peixoto

UERJ -Rio de Janeiro

.

\author{
Julia Tuñón \\ INAH -Ciudad de México \\ María de Lourdes Beldi \\ de Alcantara \\ USP-Sao Paulo \\ Mary Louise Pratt \\ NYU -Nueva York \\ Pablo Federico Semán \\ CONICET / UnSAm -Buenos Aires \\ Renato Rosaldo \\ NYU -Nueva York \\ Rose Satiko Gitirana Hikji \\ USP -Sao Paulo \\ Rossana Reguillo Cruz \\ ITESO -Guadalajara \\ Sarah Pink \\ RMIT -Melbourne
}

ENCARTES antropológicos, año 1, núm 2, septiembre 2018-marzo 2019, es una revista académica digital de acceso libre y publicación semestral editada por el Centro de Investigaciones y Estudios Superiores en Antropología Social, calle Juárez, núm. 87, Col. Tlalpan, C. P. 14000, México, D. F., Apdo. Postal 22-048, Tel. 548735 70, Fax 565555 76, encartesantropologicos@ciesas.edu.mx. Directora de la revista: Ángela Renée de la Torre Castellanos. Alojada en la dirección electrónica http://www.encartesantropologicos.mx. ISSN : 2594-2999. Las opiniones expresadas por los autores no necesariamente reflejan la postura de la revista. Se autoriza la reproducción parcial de los materiales publicados siempre y cuando se haga con fines estrictamente no comerciales y se cite la fuente. Salvo excepciones explicitadas, todo el contenido de la publicación está bajo una Licencia Creative Commons Atribución-NoComercial 4.0 Internacional. 